Urologe $2017 \cdot 56: 1-2$

DOI 10.1007/s00120-016-0278-z

Online publiziert:24. November 2016

๑) Springer Medizin Verlag Berlin 2016

CrossMark

\section{T. Maurer ${ }^{1} \cdot$ J. E. Gschwend ${ }^{1} \cdot$ M. Schwaiger ${ }^{2} \cdot$ M. Eiber ${ }^{2}$}

${ }^{1}$ Klinik und Poliklinik für Urologie, Technische Universität München, München, Deutschland

${ }^{2}$ Klinik und Poliklinik für Nuklearmedizin, Technische Universität München, München, Deutschland

\title{
Innovative Radionuklidanwendungen in der Urologie
}

In der Diagnostik und Therapie urologischer Erkrankungen sind in den letzten Jahren bahnbrechende Entwicklungen in der Radiochemie zu verzeichnen, die zu einem zunehmenden Einsatz von Radiopharmaka bei urologischen Patienten geführt haben. Dementsprechend ist die Nuklearmedizin mittlerweile sowohl in der Diagnostik als auch in der Therapie zu einem unverzichtbaren Partner der Urologie geworden. Vor diesem Hintergrund wird im vorliegenden Themenheft ein Überblick zum aktuellen Stand der Diagnostik und Therapie mit innovativen Radionuklidanwendungen gegeben. Dabei kommen gleichermaßen urologische als auch nuklearmedizinische Kollegen $\mathrm{zu}$ Wort - kennzeichnend für die Interdisziplinarität und enge Kooperation der beiden Fachdisziplinen.

Gerade die Einführung der Positronen-Emissions-Tomographie (PET) und die Kombination mit den Standardschnittbildverfahren wie der Computertomographie (CT) und seit kurzem auch der Magnetresonanztomographie (MRT) ermöglichten wesentliche Fortschritte in der Diagnostik urologischer Tumoren. Neben der rein morphologischen Information durch die CT oder MRT können nun durch die PET auch funktionelle oder metabolische Prozesse sichtbar gemacht werden. Darüber hinaus erlaubt die Kombination mit CT oder MRT eine exakte räumliche Lokalisation pathologischer Nuklidanreicherungen, sodass die PET-Hybridbildgebung mittlerweile oftmals bei der Beurteilung urologischer Tumorentitäten herangezogen wird oder sogar bereits - wie beim metastasierten
Seminom als ${ }^{18}$ F-FDG-basierte PET Einzug in Leitlinien gefunden hat. $\mathrm{Zu}-$ sätzlich erlaubt die Identifikation von neuen molekularen Zielstrukturen und die Entwicklung von spezifischen Tracern eine immer genauere Diagnostik. Und so ist es nicht verwunderlich, dass beispielsweise die gegen das prostataspezifische Membranantigen (PSMA) gerichtete PET-Diagnostik in zunehmendem Maße als „Routinediagnostik“ beim Prostatakarzinom wahrgenommen wird wie Giesel et al. beschreiben.

Gewissermaßen als direkter Brückenschlag zwischen Diagnostik und zielgerichteter Therapie sind Verfahren wie die Darstellung von Sentinel-Lymphknoten als auch die „PSMA-radioguided surgery" anzusehen. Hier wird die enge interdisziplinäre Verknüpfung zwischen Nuklearmedizin und Urologie besonders deutlich. Die Sentinel-Verfahren werden bereits seit längerem erfolgreich beim Prostata- und Peniskarzinom eingesetzt. Beim Peniskarzinom werden sie bereits als in die Leitlinien integrierter Standard angesehen. Aber auch hier gibt es neuartige innovative Weiterentwicklungen wie van der Poel et al. mit ihrem Beitrag zu Hybridtracerverfahren - also der Koppelung von radioaktiv und Fluoreszenzmarkierten Substanzen - darstellen. Das neuartige Verfahren der PSMAgezielten Chirurgie („PSMA-radioguided surgery“) erlaubt dem gegenüber intraoperativ ein direktes Auffinden von kleinen Prostatakarzinommetastasen durch radioaktive Markierung der Prostatakrebszellen selbst - gewissermaßen auf molekularer Ebene - wie Rauscher et al. erläutern.

Darüber hinaus spielen auch in der Therapie urologischer Tumoren nuklearmedizinische Verfahren eine immer bedeutendere Rolle. Erstmalig steht mit Radium-223 ein lebensverlängerndes nuklearmedizinisches Therapeutikum für Patienten mit symptomatischer ossärer Metastasierung beim Prostatakarzinom zur Verfügung. Tauber et al. fassen hier$\mathrm{zu}$ die Studienlage zusammen. Darüber hinaus wird an spezialisierten Zentren in zunehmendem Maße das PSMA aufgrund der hohen Spezifität auch als Zielstruktur für eine tumorspezifische Therapie des Prostatakarzinoms genutzt. Durch mit entsprechenden Radionukliden versehenen Liganden des PSMA können auf diese Weise onkologisch wirksame Strahlendosen direkt an die Prostatakrebszelle gebracht werden wie Heck et al. berichten. Vergleichbar mit der PSMA-basierten Diagnostik nimmt auch auf diesem Gebiet die Nuklearmedizin in Deutschland weltweit eine Vorreiterrolle ein. Aber auch bei anderen urologischen Tumorentitäten sind zielgerichtete nuklearmedizinische Therapieformen im Fokus der Forschung wie Autenrieth et al. anhand der intravesikalen Radionuklidtherapie bei Blasenkarzinompatienten nach BCGVersagen aufzeigen.

Es freut uns, Ihnen mit der vorliegenden Ausgabe von Der Urologe einige der aktuell spannendsten und innovativsten Teilgebiete der urologischen Nuklearmedizin präsentieren zu können. Wir wünschen Ihnen viel Freude beim Studieren 
der Beiträge und hoffen, dass die Lektüre dieses Heftes die zunehmende Bedeutung der interdisziplinären Zusammenarbeit beider Fächer verdeutlicht und Ihnen einen guten Überblick über aktuelle Entwicklungen verschafft.

\section{Korrespondenzadresse}

PD Dr. med. T. Maurer, FEBU
Klinik und Poliklinik für
Urologie, Technische
Universität München
Ismaninger Str. 22,
81671 München, Deutschland
t.maurer@tum.de

Interessenkonflikt. T. Maurer, J. E. Gschwend, M. Schwaiger und M. Eiber geben an, dass kein Interessenkonflikt besteht.

\section{J. Köbberling \\ Behandlungsfehler und Arzthaftung}

Praktische Hinweise für Ärzte und Patienten

Berlin: De Gruyter 2016, 128 S., 2 Abb., (ISBN: 978-3-11-047675-0), Hardcover 39,95 EUR

Das Buch Behandlungsfehler und Arzthaftung. Praktische Hinweise für Ärzte und Patienten von Johannes Köbberling (De Gruyter, 2016) gibt wichtige Praxisanleitungen zur Versachlichung des Arzt-Patienten-Verhältnisses bei vermuteten Behandlungsfehlern und ist daher ein nützlicher Leitfaden. Der Autor, Prof. Dr. Johannes Köbberling, war langjährig klinisch tätiger Internist, hat sich in vielfältigen Funktionen mit Fragen der Patientensicherheit befasst und ist $u$. a. Mitglied der Gutachterkommission für ärztliche Behandlungsfehler der Ärztekammer Nordrhein. Kritisch durchgesehen und um ein Vorwort ergänzt wurde das Buch von dem Medizinrechtler Prof. Peter Wolfgang Gaidzik. Er attestiert Köbberling, dass es inm gelungen ist, die komplexe Materie des Arzthaftungsrechts für den juristischen Laien verständlich werden zu lassen.

Wesentliches Anliegen des Buches ist das Eintreten für eine neue Kultur im Gesundheitswesen, in der Fehler nicht verleugnet oder gar vertuscht, sondern aktiv aufgegriffen werden, um daraus für die Zukunft zu lernen. Es ist sowohl als Hilfestellung für Ärzte gedacht, die sich mit Behandlungsfehlervorwürfen konfrontiert sehen, als auch als Informationsquelle und Handreichung für Patienten und Angehörige, wenn sie einen Behandlungsfehler vermuten. Dabei legt Köbberling Wert auf die Tatsache, dass Fehler zum Wesen des Menschen gehören und also auch im ärztlichen Handeln vorkommen entscheidend ist aber der Umgang damit. „Verantwortungsvolle Ärzte versuchen (...), aus ihren Fehlern zu lernen. Damit bleiben sie trotz begangenen Fehlers gute Ärzte."

(S. 1). Nach einem Kapitel über Fehlerkultur und Fehlervermeidungsstrategien widmet sich Köbberling in neun gut gegliederten und informativen Kapiteln einzelnen Aspekten des Arzthaftungsrechts: Er gibt einen Einblick in die rechtlichen Grundlagen, definiert Behandlungsfehler und ihre Ursachen, erklärt den Begriff des Schadens und des Schadensausgleichs, erörtert das Thema Beweislast und Beweiserleichterungen, erläutert ausführlich die Besonderheiten von
Diagnosefehlern, widmet sich den Themen Aufklärung, Einwilligung und Dokumentation, um schließlich auf das Stichwort Sachverständigengutachten einzugehen. Alle Kapitel werden durch zahlreiche authentische Praxisbeispiele, vornehmlich aus der eigenen Gutachtertätigkeit, ergänzt und erhalten dadurch Lebendigkeit und Anschaulichkeit. Der praktische Wert des Buches wird dadurch erhöht, dass die wichtigsten Kernsätze in farbig markierten Blöcken optisch hervorgehoben werden. Zudem beinhaltet auch das Kapitel über die Gutachterkommission unter Angabe von Adressen und Verfahrensabläufen einen konkreten Nutzwert. Was das Buch aber besonders auszeichnet, sind die beiden Schlusskapitel „Wie verhalten Sie sich als Ärztin oder Arzt bei Behandlungsfehlervorwürfen?" und "Wie gehen Sie als Patient bei möglichen Behandlungsfehlern vor?", in denen Köbberling empathisch die Perspektive des jeweiligen Betroffenen einnimmt und konkrete und konstruktive Lösungen aufzeigt.

Das Buch ermöglicht den medizinrechtlich abgesicherten Blick über den Tellerrand des klinischen Alltags, bietet konkrete Handlungsempfehlungen und ebnet den Weg in eine adäquate Fehlerkultur.

C. Güsgen (Koblenz) 